\title{
The Health and Safety of Nephrology Nurses and The Environments in Which They Work
}

\author{
Ashley Hussain* \\ Department of Health Science and Human Ecology, California State University, San Bernardino, USA
}

*Corresponding author: Ashley Hussain, Department of Health Science and Human Ecology, California State University, San Bernardino, California, USA.
Received Date: June 28, 2020

Published Date: August 27, 2020

\begin{abstract}
The articles are divided into different sections. The first section presents an introduction to the topic. The introductory part presents the background to the topic under research. Some of the important information covered under the introductory paragraph of the article includes factors such as the increase in the quality of wellbeing among nurses. Some of the factors that are mentioned in the introductory part include the quality of the nursing environment. In connection with the nursing environment factor, the article shows some of the works that have been done, which establishes the relationship between the patient's safety and the employee's safety. The introduction also provides a comprehensive meaning of the term nursing safety, based on the ideas of different authors and organizations. The second part of the article covers the methods that were used in the research. One of the methods that were used in the data collection phase includes the survey method. The main survey that was involved in the collection based on the extensive literature review of the articles related to nurse's health and safety. The article indicates that the participants in the survey were informed using different platforms such as Face book, Twitter, and RN websites. Survey Monkey was the tool that was used for data collection. The next section presents the results obtained during the study. The results indicate that there is a strong correlation between the working environments for the nurses and nurses' safety and health. The last part of the article is the conclusion of the study. The conclusion provides an overview of the results obtained during the study and the significance of the study.
\end{abstract}

Keywords: Health, Safety, Nurses, Work Environment, Culture of Safety

List of Abbreviations: AACN: American Association of Critical-Care Nurses; ANA: American Nurses Association; ANNA: The American Nephrology Nurses Association; AONE: American Organization of Nurse Executives; CDC: Center of Disease Control; NNI: Nephrology News and Issues; NOA: Nursing Organizations Alliance; RN: Registered Nurse

\section{Introduction}

The study is based on the problem that is common in a healthcare organization. In the research, the problem that is under the study concerns the relationship between the workplace environment and the wellbeing of the nurses as well as the safety of patients within a healthcare organization. For a long time, cases such as medical errors within organizations have always been linked to the poor environment created within an organization. Some of the poor working conditions for nurses include long working hours, sexual harassment, and high exposure to risks among others. This study is much concerned about such conditions in their relation to the execution of the responsibilities of the nephrology nurses.
This study examines major elements that are associated with workplace wellbeing. The study is important because it collects vital information that concerns the environment involving nurses. Basing on the issues that are identified in the study that can be used in solving the related problems to increase the safety of patients.

\section{Discussion}

\section{Methods}

The study involves a mixed method of data collection. Both the qualitative and the quantitative methods of data collection. The quantitative methods form an emphasis on objective measurement, 
and it involves the manipulation of the pre-existing data. Surveys were used as primary methods of data collection in this study. The items used in the survey were designed to meet different research techniques. To make this method of data collection more accurate, the researchers made constant references to the different works that have explored similar pieces of information. Moreover, it also involved the consultation of different nursing professionals through focus group discussions, in which the researchers asked relevant questions that they believed could help in the collection of more information. Different groups of researches mostly prefer the survey method of data collection due to its numerous advantages. The first advantage concerns the cost this method of data collection is less costly because of the online option of data collection. Again, the method is convenient because it is not subjected to manipulations and therefore the information collected through this method of data collection is accurate. Concerning this face of data collection, the timeline of surveys was enough, thus allowing more responses from different individuals to maximize the accuracy of data collected.

\section{Results and Discussions}

Under this section, a lot of information based on different elements related to wellbeing or the nursing environment was presented. Information collected based on these elements is vital in the collection of data that would help the researchers to draw a conclusion based on the correlation between the environment and the wellbeing nephrology of nurses. These elements are explored below.

\section{Satisfaction}

The results indicate that most of the individuals are satisfied with their job. According to the study findings, some were very satisfied with their jobs but others were somewhat satisfied. Basing on the level of satisfaction, the information indicates that the population of very satisfied individuals is almost equal to the population of the somewhat satisfied individuals. Still, under satisfaction, the information indicates that most of the RN nurses would definitely recommend pursuing the nursing concerns and the same population would probably recommend. Regarding where the job satisfaction is high; probably elements such as regular appreciations must be present within an organization. Such elements are highly dependent on the organizations' Human resource management.

Considering the significance of motivation within the workplace, positive comments and recognition are major players that influence the level of motivation among workers. From the information, it is evident that RNs have different levels of satisfaction because they belong to different kinds of organizations. Those nurses that represent highly satisfied employees probably come from organizations that consider vital factors such as workplace reinforcement through positive comments, [1]. On the other hand, the proportions of nurses who are somewhat satisfied with their jobs belong to organizations which in one way or the other have some qualities that do not support workplace wellbeing. Lastly, the possible reason why most of the RNs would recommend pursuing a nursing career because of the high demand of the nurses on the job market as compared to other professions.

\section{Mental and physical abuse}

All forms of abuse are among the major factors that contribute to unhealthy working conditions such as psychological stress and depression [2]. Therefore, enacting policies related to workplace forms of abuse is one of the recommendations that can be used to promote a healthy workplace. The research identifies different forms of abuse in the workplace with sexual harassment being the most dominant factor among the nurses and the patients. Other forms of harassment include verbal and physical harassment. Regarding physical and mental abuse, it is upon the organization to establish policies that will prevent any form of abuse [3]. The most common policy among many organizations is zero-tolerance to all forms of abuse.

However, the information indicates the there are other instances where abuse is never reported at all. The study found that a high percentage of the organizations claim to have zero-tolerance to all forms of abuse within their organizations, but that is not the case. The zero-tolerance policy is essential in an organization setting as it protects the wellbeing of the nurses working in an organization. Most of the workers are not aware of the existence of policies that protect their wellbeing in an organization, thus end up suffering in silence. Therefore, it is the responsibility of organizations to provide employees with a good working environment that is free from all forms of abuse. Giving the nurses' needs an appropriate rank on the Maslow's hierarchy of human needs can improve the efficiency of an organization. To be more specific, security needs should be prioritized to enhance a safer healthcare facility [4]. By so doing, the employees will be in a better position to work hard to reach the self-actualization.

\section{Long, intense hours and fatigue}

Long hours on shift are one of the major causes of inefficiencies at work, [5]. The information under this study indicates that nurses work for longer hours. The study found that overtime at work is considered a voluntary activity but organizations treat it as a requirement for the nurses. It is arguable that overworking nurses not only exposes them to health-related problems but also increases the chances of occurrence of medical errors. [6] Listed some of the dangers that are associated with long working hours. Long shifts put the nurses at the risk of contracting cardiac diseases. They also establish that diseases such as hypertension and work-related stress disorders are among the common risk that individuals working long hours are exposed to. 


\section{Leadership}

Leadership is one of the determinants of the working environment safety. In many organizations, poor leadership contributes to a high rate of turnover. Within the healthcare setting, the study found that the type of leadership greatly influences both the patient and the nurses' wellbeing either directly or indirectly, [7]. For instance, a manager with good leadership skills will understand the effects of motivation among employees and the need to create a motivational environment. Once the leader understands that, then he or she will find ways to motivate the nurse who will then put the needs of patients before everything. The research done by [8] gives an excellent idea on working environments with poor leadership skills. They say that leadership skills have room for adjustment. This idea gives a clue about the significance of training programs within an organization. The introduction of training programs within an organization is one of the crucial steps that an organization can take to improve the wellbeing of the individuals working within a given facility.

\section{Staffing and work that gets done}

Having enough number of staff is one of the major factors that help in the reduction of healthcare-related problems. Some of the major indicators of rightful staffing include a reduction in nursing fatigue. This is because the high number of nurses will obviously reduce cases of long working hours and overtime. A rightful patient-nurse ratio also reduces cases that are usually associated with medical errors, which leads to high mortality rates. Therefore, if mortality rates are low, it means that the facility is equipped with the right number of nurses, [9]. According to the research [10], several negative outcomes are associated with the unbalanced patient to RN ratio in the dialysis treatments. Some of the negative impacts such a ration in dialysis treatments include skipping of the treatments, and increase in the cases of dialysis hypertension among others. Generally, it is clear that the high number of patients being attended to by a single nurse, the high the number of medical errors and therefore increasing risks on patient's safety. This information, therefore, provides an important factor that should be examined in instances where there are high frequencies of medical errors.

\section{Conclusion}

The results section presents some of the vital factors that should be considered to heighten safety in healthcare units. Considering satisfaction, it is evident that job satisfaction is the major determent of worker's retention. Another factor that determines workplace safety is security and this is based on Maslow's hierarchy of human needs. Some of the main factors that contribute to unhealthy working conditions as indicated in the article include both physical and mental abuse. However, it is indicated that zero-tolerance is the main policy that helps in the reduction of cases of abuse. Leadership is another factor; leadership, as presented in the article, has two faces, good leadership, and poor leadership. The study indicates that good leadership provides a healthy working environment for the nurses and bad leadership might interfere with the working conditions. However, the study found that leadership can be adjusted through training. Lastly, rightful staffing provides good working conditions and increase the level of patient safety. The high patient-RNs ratio is prone to medical errors that may lead to death. It is therefore acceptable to say that factors explored under this study are vital in the promotion of RN wellbeing and which in turn increases patient safety.

\section{Acknowledgement}

I would like to express my deep gratitude to Ulrich, Beth T., and Tamara M. Kear for their research which provided most of the information for my mini research. Finally, I wish to thank my parents and loving husband for their support and encouragement throughout my study.

\section{Conflict of Interests}

Author declares no conflicts of interests.

\section{References}

1. Baljoon RA, Banjar HE, Banakhar MA (2018) Nurses work motivation and the factors affecting it: a scoping review. International Journal of Nursing \& Clinical Practices.

2. Ahmed A S (2012) Verbal and physical abuse against Jordanian nurses in the work environment. East Mediterr Health J 18(4): 318-324.

3. Becton JB, Gilstrap JB, Forsyth M (2017) Preventing and correcting workplace harassment: Guidelines for employers. Business Horizons 60(1): 101-111.

4. Maslow AH (1954) Motivation and personality. New York. NY: Harper.

5. Virtanen M, Kivimäki M (2018) Long working hours and risk of cardiovascular disease. Current Cardiol Rep 20(11): 123.

6. Wong K, Chan AH, Ngan SC (2019) The effect of long working hours and overtime on occupational health: a meta-analysis of evidence from 1998 to 2018. Int J Environ Res Public Health 16(12): 2102.

7. Press Ganey (2017) 2017 Press Ganey nursing special report: The influence of nurse manager leadership on patient and nurse outcomes and the mediating effects of the nurse work environment. South Bend, IN: Press Ganey.

8. Blake N, Leach LS, Robbins W, Pike N, Needleman J (2013) Healthy work environments and staff nurse retention: The relationship between communication, collaboration, and leadership in the pediatric intensive care unit. Nurs Adm Q 37(4): 356-370.

9. Blegen M, Goode C, Spetz J, Vaughn T, Park S (2011) Nurse Staffing Effects on Patient Outcomes: safety-net and non-safety-net hospitals. Med Care 49(4): 406-414.

10. Thomas Hawkins C, Flynn L, Clarke SP (2008) Relationships between registered nurse staffing, processes of nursing care, and nurse-reported patient outcomes in chronic hemo dialysis units. Nephrology Nursing Journal, 35(2): 123-130, 145. 